\title{
Non-Linear and Non-Conventional Speech Processing: Alternative Techniques
}

\author{
Jordi Solé-Casals • Vladimir Zaiats • \\ Enric Monte-Moreno
}

Received: 8 August 2010/ Accepted: 12 August 2010/Published online: 26 August 2010

(C) Springer Science+Business Media, LLC 2010

This special issue aims to cover some problems related to non-linear and non-conventional speech processing. The origin of this volume is in the ISCA Tutorial and Research Workshop on Non-Linear Speech Processing, NOLISP'09, held at the Universitat de Vic (Catalonia, Spain) on June 25-27, 2009. The series of NOLISP workshops started in 2003 has become a biannual event whose aim is to discuss alternative techniques for speech processing that, in a sense, do not fit into mainstream approaches. A selected choice of papers based on the presentations delivered at NOLISP'09 has given rise to this issue of Cognitive Computation.

The papers hereinafter deal with the following topics:

What would happen if we were able to overcome the limitations of audiovisual human systems? The answer could be found in the paper by V. Espinosa-Duró, M. Faundez-Zanuy, and J. Mekyska.

A growing recurrent self-organizing model for phoneme recognition is presented by Ch. Jlassi, N. Arous, and N. Ellouze.

J. Solé-Casals $(\bowtie) \cdot$ V. Zaiats

Department of Digital Technologies and Information, Escola Politècnica Superior, Universitat de Vic, c/. de la Laura, 13, 08500 Vic (Barcelona), Spain e-mail: jordi.sole@uvic.cat

V. Zaiats

e-mail: vladimir.zaiats@uvic.cat

E. Monte-Moreno

Department of Signal Theory and Communications, Escola Tècnica Superior d'Enginyeria de Telecomunicació de Barcelona, Universitat Politècnica de Catalunya, c/. Jordi Girona 1-3, Campus Nord UPC, Edifici D5, 08034 Barcelona, Spain e-mail: enric.monte@upc.edu
An algorithm for voiced/unvoiced decision and pitch estimation from speech signals based on classification of peaks using the auto-correlation of the speech multi-scale product is presented by M. A. Ben Massoud, A. Bouzid, and N. Elloze.

A feature reduction system based on the Discriminative Common Vector is proposed by C.M. Travieso, M. del Pozo, M.A. Ferrer, and J. B. Alonso.

A new approach to the diagnosis of Ataxia SCA-2 by application of independent component analysis to a set of data obtained by electro-oculography is presented by R. V. García, F. Rojas, C. G. Puntonet, B. San Román, L. Velázquez, and R. Rodríguez.

A formant tracking technique based on Fourier ridges detection aiming at improving the performance of these algorithms is presented by I. Jemaa, K. Ouni and Y. Laprie

A new architecture for vocabulary independent keyword detection for cognitive virtual agents (SEMAINE system) is proposed by M. Wöllmer, F. Eyber, A. Graves, B. Schuller, and G. Rigoll. Their word spotting model is composed of a dynamic-Bayesian network and a bidirectional long-short-term memory recurrent neural network.

An insight into non-linear transformations for improving the performance of an entropy-based voice activity detector is made in the paper by two guest editors (J. S. and V.Z.).

By no means has the range of topics in this Special Issue covered all directions in non-linear speech processing. We would rather consider it a sample presenting several areas in progress. In any case, we hope that they will be of interest for the readers of Cognitive Computation.

Our thanks are due to the Editor-in-Chief, Dr. Amir Hussain, for permanent support in preparation of this issue. Of course, this issue would have been impossible without the contributions of all authors. On the other hand, anonymous referees have also done a great job by carefully 
reading the papers and enriching them by their comments and remarks.

It is our pleasure to gratefully acknowledge the financial support obtained by NOLISP'09 from the Ministerio de
Ciencia e Innovación of Spain (grant TEC2008-02717-E/ TEC), from the Universitat de Vic, ISCA, and EURASIP. 